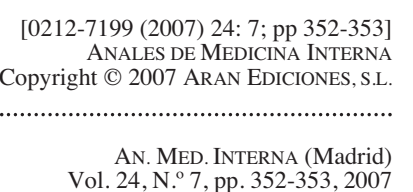

Vol. 24, N. ${ }^{\circ}$ 7, pp. 352-353, 2007

\title{
¿Es el fango un antiinflamatorio?
}

\author{
M. I. GIACOMINO, D. F. DE MICHELE \\ Departamento de Postgrado. Facultad de Ciencias de la Salud. Universidad Nacional de \\ Entre Ríos. Argentina
}

\author{
IS MUD AN ANTI-INFLAMMATORY?
}

\section{RESUMEN}

Es dificultoso hallar una herramienta terapéutica utilizada por 25 siglos; el fango es una de ellas.

El empirismo remoto -no científico por cierto- permite al menos alguna clase de inferencias; si la acción de los fangos sobre el proceso inflamatorio fuera neutra o nula, lo sabríamos ya.

Los fangos tienen un lugar como tratamiento no farmacológico en ciertas situaciones clínicas como procesos articulares degenerativos, desordenes cutáneos, etc. Los babilonios conocían sus efectos. Las primeras explicaciones científicas en relación a los efectos de los fangos han sido comunicadas en los últimos años por algunos investigadores.

La fangoterapia en pacientes con artritis, parece provocar variaciones en los niveles de algunos aminoácidos involucrados en la homeostasis del cartílago, así como reducción en los índices de dolor en gonartrosis.

También altera los niveles séricos de óxido nitroso, mieloperoxidasa y glutationperoxidasa en pacientes con artritis, y es capaz en pacientes afectados de artritis, de modificar los niveles de beta endorfinas y hormonas del estrés, por reducción de la inflamación y el dolor, disminuyendo así las causas del estrés.

Otros autores confirman que el stress termal asociado a la fangoterapia activa la pituitaria y que el efecto bioquímico de los componentes del fango, pueden separarse de su acción física por el calor. Más aún, se ha comunicado actividad esteroidea y antimicrobiana en ciertos fangos terapéuticos.

PALABRAS CLAVE: Fango. Peloides. Fangoterapia.

\section{ABSTRACT}

It's really hard to find a therapeutic tool used along 25 centuries; mud certainly is one.

The remote empirism -not actually scientific-allows at least some kind of inferences; if the action of mud over the inflammatory process were neutral or harmful we should knew it.

Mud has a place as a non-pharmacological tool in certain clinical settings, such as degenerative articular processes, skin disorders, and others. Babylonians knew its effects. Former scientific explanations regarding the mud action have been communicated in last years by some researchers.

Fangotherapy in arthritis patients seems to cause variations in amino acid involved in cartilage homeostasis, and also produce reduction in pain ratings in gonarthrosis. Mud modifies nitric oxide, myeloperoxidase and glutathione peroxidase serum levels in arthritic patients and beta-endorphin and stress hormones in patients affected by osteoarthritis by reducing inflammation, pain and therefore diminishes the cause of stress. Has been confirmed that the thermal stress associated with Fangotherapy, activates the pituitary gland and the biochemical effects of peat components have aside from their physical-thermal effects. Furthermore, steroids and antimicrobial activity of certain therapeutic mud has been suggested.

KEY WORDS: Mud. Peloid. Pelotherapy. Fangotherapy. Mud Baths. Mud Bath. Peat Therapy.

Giacomino MI, De Michele DF. ¿Es el fango un antiinflamatorio?. An Med Interna (Madrid) 2007; 24: $352-353$.

En la medicina de hoy, las conclusiones de las pruebas clínicas realizadas sobre grandes series de pacientes, y en varios centros de mundo al mismo tiempo, dominan y dirigen el rumbo de la terapéutica. La intervención de la industria, (farmacéutica y/o proveedora de tecnología médica), suele ser un impulso potente en el diseño de este tipo de estudios, que nos abruman con sus conclusiones, su perfil global, su volumen, los centros de referencia participantes y las personalidades científicas involucradas.
Recientemente algunos grupos de investigadores se han interesado en la explicación de un fenómeno aprovechado por el hombre desde hace más de 25 siglos: la utilización del fango como antinflamatorio.

Hasta donde sabemos, existe poca o ninguna financiación para desarrollar líneas de investigación, por lo que hay contados grupos de trabajo que llevan adelante iniciativas en esta dirección. Su estudio y uso terapéutico se halla restringido a centros específicos, especialmente en Europa y Cuba. 
Algunos fármacos que debieron retirarse de circulación, mostraron su eficacia en ensayos científicos multicéntricos, pero no lograron superar la "prueba del tiempo", y a pocos años de su lanzamiento, cuando su uso fue masivo, supimos que tenían algún efecto potencialmente dañino para la salud humana.

Del empirismo remoto, puede extraerse un indicador que suele ser infalible; la extensión de tiempo en que se ha utilizado la herramienta terapéutica que se esta evaluando,

La fangoterapia en la enfermedad articular degenerativa, es una modalidad terapéutica generalmente auto indicada, probada y utilizada más de 2.500 años por millones de personas.

El empirismo remoto - por cierto, no científico- al menos permite inducir que si la acción de las aguas minerales termales y los fangos sobre la inflamación articular fuera neutra o nociva, después de 2.500 años, ya lo sabríamos.

Se ha repetido que en las enfermedades degenerativas articulares, el beneficio del fango se debe al calor y no al material aplicado en sí mismo. En realidad, apenas sabemos por qué ocurre la acción antinflamatoria relatada por los propios pacientes -si es que ocurre- $y$ es posible que nunca lo sepamos completamente.

Conocemos en cambio que desde siempre, el hombre primitivo, utilizó componentes de su hábitat, para remediar sus dolencias, especialmente del suelo, como sales, plantas, hongos, mohos, frutos, surgentes de aguas, minerales, algas, flores, barro, etc.

La propia medicina moderna lo ha hecho con éxito.

Algunos investigadores, están aportando en estos últimos años datos que constituyen las primeras explicaciones científicas a un hecho que los legos conocen desde hace siglos.

Básicamente, empieza a entenderse que la exposición del cuerpo humano a fluidos termales (agua, vapor) y fangos minerales, produce fenómenos metabólicos y sistémicos, más allá de su acción local, habitualmente reconocida.

En el 2004 ha sido comunicado que el fango utilizado en artrosis produce elevación sérica de algunos aminoácidos como el triptofano, cisteína y citrulina (1), y que en gonartrosis ayuda a controlar el dolor (2).

Ha sido también reportado, que tras la terapia con fango en artrosis, pueden registrarse modificaciones beneficiosas en algunos mediadores de la homeostasis del cartílago, como la mieloperoxidasa y el óxido nitroso (3).

\section{Bibliografía}

1. Bagnato G. Clinical improvement and serum amino acid levels after mud-bath therapy. Int J Clin Pharmacol Res 2004; 24: 39-47.

2. Wigler I. Spa therapy for gonarthrosis: A prospective study. Rheumatol Int 1995; 15: 65-8.

3. Bellometti S. Mud bath therapy influences nitric oxide, myeloperoxidase and glutathione peroxidase serum levels in arthritic patients. Int $\mathbf{J}$ Clin Pharmacol Res 2000; 20: 69-80.

4. Pizzoferrato A. Beta-endorphin and stress hormones in patients affected by osteoarthritis undergoing thermal mud therapy. Minerva Med 2000; 91: 239-45.

5. Giusti P. Stress hormones liberated by fangotherapy. ACTH and betaendorphin levels under heat stress. Fortschr Med 1990; 108: 601-3.

6. Beer A. The effect of peat components on endocrine and immunological parameters and on trace elements-results of two pilot studies. Clin Lab 2001; 47: 161-7.

7. Aquino R. The presence of steroid compounds in therapeutic muds. Boll Soc Ital Biol Sper 1985; 61: 1261-6.

8. Ma'or, Z. Antimicrobial properties of Dead Sea black mineral mud. Int J Dermatol 2006; 45: 504-11.

9. Tarkhan-Muuravi ID, Dzhakobiia NV. Effect of complex rehabilitation by
Otros importantes mediadores de la inflamación incluyendo endorfinas y hormonas de estrés, parecen alterar sus valores plasmáticos en pacientes con artrosis que utilizan fangos (4-5).

El efecto de la turba ha podido separarse de su acción térmica, y se ha sugerido que se debería a los metales pesados que la componen (plomo, manganeso, cadmio y cobre).

Investigadores europeos comunican elevaciones del estradiol en mujeres post menopáusicas, tras el uso estos fangos ricos en metales pesados (6).

El probable contenido de esteroides en el fango, ha sido objeto de comunicaciones (7) y recientemente se ha sugerido que ciertos fangos hipersalinos (Mar Muerto) poseen actividad bactericida (8).

La acción antinflamatoria de los fangos, también ha sido recientemente sugerida por Tarkhan-Muuravi (2006) cuando examina el resultado de su aplicación a pacientes con trauma de troncos nerviosos en la rehabilitación de neuroapraxia y axonotmesis (9).

El efecto antinflamatorio de los peloides, ha podido reproducirse en el laboratorio, en animales de experimentación con artritis inducida (10), y se ha sugerido recientemente que modificaciones en la actividad de neutrófilos y macrófagos intervinientes en el proceso inflamatorio, pudieran tener algún lugar en esta acción (11).

Estas propiedades antiflogísticas de ciertos fangos han sido probadas en enfermedades articulares como la artritis reumatoidea $(12)$, osteoartritis $(13,14)$ así como en la espondilitis anquilosante (15).

Se conocía de la existencia de alguna de estas acciones de los fangos, pero hasta hace poco, no podíamos aportar información sobre su intimidad fisiopatológica.

La investigación ha comenzado a echar luz sobre estos fenómenos aumentando nuestro conocimiento sobre los mismos en los últimos años.

Es probable que además de las acciones de los fangos comentadas y comunicadas, su aplicación en un ambiente de tranquilidad y paz, el paisaje en armonía, el relax y el ocio que rodean a la fangoterapia, pudieran tener un lugar no menor en los resultados que se reportan, potenciando su efecto placebo. physical factors (therapeutic mud, waves of millimeter range) on the indices of inflammation process and immune status in patients with traumas of peripheral nervous system. Georgian Med News 2006; 132: 72-6.

10. Cozzi F, Carrara M, Sfriso P, Todesco S, Cima L. Anti-inflammatory effect of mud-bath applications on adjuvant arthritis in rats. Clin Exp Rheumatol 2004; 22: 763-6.

11. Starichkov AA, Bondareva ZG. The role of leukocytes in mechanism of action of balneopeloidotherapy. Vopr Kurortol Fizioter Lech Fiz Kult. 2004; 5: 29-31.

12. Codish S, Abu-Shakra M, Flusser D, Friger M, Sukenik S. Mud compress therapy for the hands of patients with rheumatoid arthritis. Rheumatol Int 2005; 25: 49-54. Epub 2003; 14.

13. Grassi M, Lucchetta MC, Rini GB, Raffa S. Fangotherapy in chronic degenerative rheumatic diseases. Clin Ter 2003; 154: 45-8.

14. Grigor'eva VD, Orus-ool VK, Fedorova NE. Low-temperature peloids in rehabilitating osteoarthritis Patients. Vopr Kurortol Fizioter Lech Fiz Kult 2001; 8-11.

15. Barnatskii VV. Effects of radon and peloid therapy on functional condition and quality of life in patients with seronegative spondyloarthritis Vopr Kurortol Fizioter Lech Fiz Kult 2005; 3: 26-30. 